medical complexity of patients of this population, as defined by the number medical diagnoses, impacts the type of treatment rendered as well as how frequently these patients are seen for dental care (rate of appointments). DISCUSSION/SIGNIFICANCE OF FINDINGS: Over- or under-utilization of dental treatment modalities can impact the oral health status and outcomes for patients with special needs. The differences in types and frequency/ rate of dental treatment rendered for patients of different medical complexity can further impede their oral health and systemic health status and health outcomes.

16306

\section{Genomic epidemiology of SARS-CoV-2 across New Mexico and the Mountain West}

Daryl Domman ${ }^{1}$, Kurt Schwalm ${ }^{1}$, Twila Kunde ${ }^{2}$, Joseph Hicks ${ }^{2}$, Michael Edwards ${ }^{2}$, Noah Hull ${ }^{3}$, Wanda Manley ${ }^{3}$, Ethan RomeroSeverson ${ }^{4}$, Emma Goldberg and Darrell Dinwiddie ${ }^{1}$

${ }^{1}$ University of New Mexico Health Sciences Center; ${ }^{2}$ New Mexico Department of Health; ${ }^{3}$ Wyoming Public Health Laboratory; ${ }^{4}$ Los Alamos National Laboratory

ABSTRACT IMPACT: Genomic data can be used by policy and decision makers to guide, and assess the impact of, public health responses to the COVID-19 pandemic. OBJECTIVES/GOALS: Our objective is to investigate the transmission and population dynamics of SARS-CoV-2 in New Mexico and other Mountain West states using whole genome sequencing. Understanding how the virus is spreading within and between communities is vital for the design of rational, evidence-based control measures. METHODS/STUDY POPULATION: We obtained an aliquot of $500 \mathrm{ul}-1 \mathrm{ml}$ of inactivated viral transport media (VTM) from positive SARS-CoV-2 nasopharyngeal swabs as determined by qPCR from the New Mexico Department of Health, TriCore Reference Laboratory, Idaho Bureau of Laboratories, and Wyoming Public Health Laboratory. We extracted viral RNA from the VTM, and sequenced the genomes using the methodology as described by the widely adopted ARTIC amplicon tiling protocol for SARS$\mathrm{CoV}-2$. Viral genomes were then sequenced on either an Illumina MiSeq or an Oxford Nanopore Technologies (ONT) GridION. We placed these samples within the context of globally representative sequences made available via the GISAID database. Consensus sequences were aligned and added into this global dataset using the Nextstrain augur pipeline. RESULTS/ANTICIPATED RESULTS: We sequenced over 1,000 SARS-CoV-2 genomes thus far from New Mexico $(n=861)$, Wyoming $(n=213)$ and Idaho $(n=44)$. We used this sequence data to infer the transmission dynamics and spread of the virus, both within states and in context of regional and international spread. We inferred at least 128 separate introductions of the virus into New Mexico and at least 29 introductions into Wyoming. The origins of these introductions are diverse, spread across multiple regions in the US and abroad. We also sequenced samples from an individual who had multiple positive tests over time. Our results suggest that this individual was reinfected with a different strain than that of the initial infection. DISCUSSION/SIGNIFICANCE OF FINDINGS: Our data show that New Mexico and other Mountain West states have continually experienced many introductions of the virus that then seed local outbreaks. By understanding the number of introductions over time, we can assess the impact of travel restrictions on transmission. Our data also supports that some individuals can be re-infected.

\section{Describing Physical Symptoms among Patients with PTSD at an Anxiety Clinic in Puerto Rico}

Marie Torres-Valentin ${ }^{1}$, Karen G. Martinez-Gonzalez $z^{1}$ and Alfonso Martinez-Taboas ${ }^{2}$

${ }^{1}$ University of Puerto Rico, Medical Sciences Campus;

${ }^{2}$ Interamerican University of Puerto Rico, Metropolitan Campus

ABSTRACT IMPACT: Our work will provide valuable information about the associations between physical symptoms and PTSD in patients from a Spanish-speaking, evidence-based clinic. OBJECTIVES/GOALS: In this reserach study, we want to describe physical symptoms of patients with a preliminary PTSD diagnosis. We also want to explain associations between physical symptoms, and the presence, or absence of PTSD, and to evaluate findings in terms of prevention services, referrals, and alternatives for augmenting treatment-adherence. METHODS/STUDY POPULATION: This was a descriptive, secondary database analysis of the Center for the Study of Fear and Anxiety (by its Spanish acronym, CETMA). The database included information of the initial evaluation between 2012 and 2019. We aimed to describe sociodemographic and medical variables, and evaluate associations, in terms of the presence or absence of PTSD. RESULTS/ ANTICIPATED RESULTS: Patients with PTSD were mostly women, single, with a completed bachelor's degree. The majority had at least one neurological, or musculoskeletal condition. Respiratory conditions were the least represented. We found significant associations between musculoskeletal, neurological, and ear/ nose/throat conditions, in terms of PTSD diagnosis. DISCUSSION/SIGNIFICANCE OF FINDINGS: Puerto Rico recently experienced two hurricanes, several earthquakes, and the pandemic. Findings provide data about the interface between mental and physical symptoms of patients with PTSD. We recommend a randomized population study with mental and physical variables, for understanding possible effects of cumulative stress in Puerto Ricans.

38351

\section{Immune-related adverse events in cancer patients receiving immune checkpoint inhibitors*}

Margaret Byrne, Mathew Lucas, Lori Pai, Janis Breeze and Susan Parsons

Tufts Medical Center

ABSTRACT IMPACT: The existing literature describing immunerelated adverse events (irAE) has predominantly focused on clinical trial populations, which may not be representative of the broader population receiving immune checkpoint inhibitors (ICI), so we sought to perform a comprehensive evaluation of irAE in a realworld population of cancer patients being treated with ICI. OBJECTIVES/GOALS: With a cohort of patients with malignancy treated with ICI, we characterized incidence, severity, timecourse of ir-AE. We sought to inform providers who prescribe ICI to recognize the clinical burden of irAE in an effort to more effectively communicate the benefits and risks of ICI with patients. METHODS/STUDY POPULATION: After obtaining approval from the institutional review board, we used a pharmacy database to identify adult cancer patients treated with an ICI between January 2014 and October 2018. We used electronic medical records to obtain 
baseline variables. Each patient was followed at each clinic visit for 12 months for development of a physician-reported irAE.

For irAE, site and grade were recorded as documented by the provider. At diagnosis and each follow-up visit, we collected: 1) adjustments to ICI, immunosuppression, and hormone therapy.

Continuous variables were summarized using mean and standard deviation (SD) or median and interquartile range (IQR). Categorical variables were summarized using frequencies and percentages. Time to development and resolution of irAE were calculated using Kaplan-Meier curves. RESULTS/ANTICIPATED RESULTS: Among 131 patients, two-thirds were men, and 60\% were Caucasian with a mean age was 65 years. Nearly $40 \%$ had an Eastern Cooperative Oncology Group performance score of 2 or higher. A small proportion $(3.1 \%)$ had an autoimmune disorder. Nearly half had lung cancer $(49.6 \%)$, and several had received radiation (33.6\%). Over $70 \%$ were former or current smokers. In total, 57 patients (43.5\%) developed an irAE, resultng in a total of 95 irAE, at a median of 208 days (126 days, not reached). The most common irAE included dermatitis, thyroiditis, pneumonitis, and hepatitis. Of $95 \mathrm{irAE}$, half were grade $1,30 \%$ were grade 2 , and nearly $20 \%$ were grade 3 or higher. Median time to resolution was 85 days (56-183 days). DISCUSSION/SIGNIFICANCE OF FINDINGS: This study demonstrates that irAE are clinically impactful in this relatively unfit, medically complex population, which may be a more accurate reflection of patients receiving ICI in everyday practice. This study showcases that this population is susceptible to irAE, although this needs to be examined in larger, prospective trials.

55715

\section{Quantification of Neonatal THC Exposure Following Prenatal Marijuana Use}

Stefanie Kennon-McGill, Ph.D., Heather Moody, Jeff Moran, Ph.D., and Laura James, MD

University of Arkansas for Medical Sciences

ABSTRACT IMPACT: Quantification of neonatal THC exposure will allow for better insight into how THC exposure correlates with neurodevelopmental outcomes. OBJECTIVES/GOALS: Tetrahydrocannabinol (THC) use has become increasingly prevalent in recent years, including among pregnant women. However, few, if any, clinical studies have quantified precise in utero exposure levels of THC during pregnancy. Our study aims to fill this gap by using analytical methods to quantify THC in mother and baby following prenatal THC use. METHODS/STUDY POPULATION: Pregnant women were asked to give a self-report of all cannabis and cannabinoid use during pregnancy, including dose, frequency, and route of consumption. Upon arrival at the labor and delivery unit, maternal blood samples were collected. Immediately following birth and 24 hours after birth, umbilical cord and neonatal blood samples were collected, respectively. All blood samples were analyzed using tandem liquid chromatography-mass spectrometry (LC-MS) for the presence of THC, tetrahydrocannabinol carboxylic acid (THC$\mathrm{COOH})$, and hydroxy-tetrahydrocannabinol (THC-OH). Maternal THC and metabolite levels were compared to both cord and neonate samples. RESULTS/ANTICIPATED RESULTS: To date, we have collected 3 mother-infant sample dyads and 4 mother-infant control samples. We anticipate collecting a total of 20 mother-infant samples from each group. We will quantify levels of THC and its metabolites in maternal samples and compare these to cord and infant samples.
We expect that THC/metabolite levels will vary as a function of dose and frequency of consumption. We also expect that THC/metabolites will be higher in umbilical cord blood relative to neonatal blood. DISCUSSION/SIGNIFICANCE OF FINDINGS: This study is among the first to directly measure exposure in the neonate following prenatal cannabis use. Quantification of THC/metabolite concentrations will be supplemented with developmental evaluations of infants at 6 and 12 months of age in order to gain better insight into how THC exposure correlates with neurodevelopmental outcomes.

73662

Racial Disparities in Septic Shock Mortality: Results from the OneFlorida Data Trust Cohort

Lauren Page Black, MD, MPH, University of Florida College of Medicine - Jacksonville; Charlotte Hopson, MS, University of Florida College of Medicine - Jacksonville, Center for Data

Solutions; Elizabeth DeVos, MD, MPH, University of Florida College of Medicine - Jacksonville; Michael Puskarich, MD, MS, University of Minnesota; Rosemarie Fernandez, MD, University of Florida College of Medicine; Faheem Guirgis, MD, University of Florida College of Medicine - Jacksonville; Cynthia Garvan, PhD, University of Florida College of Medicine

ABSTRACT IMPACT: Identifying racial disparities in septic shock mortality, a common and lethal condition, can inform future research and policy efforts aimed at understanding the drivers these disparities and addressing the underlying factors in order to reduce disparities and improve health. OBJECTIVES/GOALS: Septic shock is a major public health problem with significant mortality. Existing data indicate racial disparities in sepsis incidence, but evidence is limited on differences in septic shock outcomes. Our objective was to determine the association between race and septic shock mortality in a statewide cohort while controlling for clinical factors. METHODS/STUDY POPULATION: This was a retrospective analysis of septic shock patients in the One Florida Data Trust between 2012-18. Data was collected regarding age, sex, race, insurance status, and selected comorbid conditions [liver disease, hypertension, chronic obstructive pulmonary disease (COPD), congestive heart failure $(\mathrm{CHF})$, end-stage renal disease (ESRD), and human immunodeficiency virus infection (HIV)]. To account for severity of illness, we assigned Sequential Organ Failure Assessment scores for components based on laboratory values (labSOFA), and collected data on mechanical ventilation use and initial lactate.

The primary outcome was 90-day mortality. The Least Absolute Shrinkage and Selection Operator (LASSO) method was used for variable selection for the multivariable regression model. RESULTS/ANTICIPATED RESULTS: There were 13,932 septic shock patients with a mean (SD) age of 61(16) years. Of these, $68 \%$ identified as white, $28 \%$ as black, $2.1 \%$ as Hispanic, and $2.0 \%$ as other races. 90-day mortality was $32 \%(n=4,437)$ and $59 \%$ required mechanical ventilation. Significant independent predictors of mortality in the regression model were age (OR 1.04; $\mathrm{p}<0.01$ ), black race $(1.72 ; \mathrm{p}<0.01)$, lactate $(1.10 ; \mathrm{p}<0.01)$, mechanical ventilation $(3.62 ; \mathrm{p}<0.01)$, labSOFA $(1.18 ; \mathrm{p}<0.01)$, history of liver disease $(1.75 ; \mathrm{p}<0.01)$, hypertension $(0.70 ; \mathrm{p}<0.01)$, COPD $(0.87 ; \mathrm{p}<0.01)$, $\operatorname{CHF}(1.18 ; \mathrm{p}<0.01), \operatorname{HIV}(1.30 ; \mathrm{p}=0.05)$, and the interaction between age and black race. Black patients had 1.72 times the odds of mortality compared to white patients. For every one-year decrease in age, 